\title{
Wilson Disease Mutations in the American Population: Identification of Five Novel Mutations in ATP7B
}

\author{
Danadevi Kuppala ${ }^{1}$, Jie Deng ${ }^{2}$, George J. Brewer ${ }^{3,4}$, Michael M. Wang ${ }^{1,5}$ and Jimo Borjigin ${ }^{*}, 1$ \\ Departments of ${ }^{1}$ Molecular and Integrative Physiology; ${ }^{2}$ Department of Medicine; ${ }^{3}$ Human Genetics; ${ }^{4}$ Internal \\ Medicine, and Neurology, University of Michigan Medical School, Ann Arbor, MI 48109-5622; Johns Hopkins \\ University School of Medicine, Baltimore, MD 21205, USA
}

\begin{abstract}
Wilson disease is an autosomal recessive disorder characterized by toxic accumulation of copper in a number of organs such as liver and brain, which results in significant disability or death if left untreated. Wilson disease is caused by mutations in ATP7B, a copper transporter. We analyzed 108 American Wilson disease patients, who are predominantly White, for mutations in ATP7B. Consistent with studies from other populations, H1069Q was the most common mutation in this group of patients, accounting for $40.3 \%$ of the sequenced alleles; 26 of the 108 patients were homozygous, and 35 patients were heterozygous for this mutation. We also identified 24 additional ATP7B mutations, of which five were novel. The five new mutations consist of two insertion mutations (2302insT and 3843insT), one splice site mutation (IVS11+2:T>A), one combination of deletion (2bp) and insertion (19bp) (3693-3697delins19bp), and one missense mutation (G1213S). All variants are predicted to be disease-causing mutations. Ninety six percent of all mutations we identified were clustered in regions encoding the C-terminal half (catalytic domain) of the ATP7B protein. Furthermore, we found that $84 \%$ of the mutant alleles identified in the American population are located in exons 14 and 18 .
\end{abstract}

\section{INTRODUCTION}

Wilson disease (WD) is an autosomal recessive disorder of copper transport with a world-wide prevalence of 30 per million [1-3]. WD is associated with decreased incorporation of copper into ceruloplasmin and a buildup of copper in target tissues, which result in hepatocellular failure and/or basal ganglia degeneration $[2,4]$. This disorder is one of a few hepatic or neurological disorders that is treatable [1, 2, 4]. Thus, identification of genetic mutations for suspected WD patients may play a critical role in successful treatment.

The WD gene ATP7B, comprised of 21 exons spanning $80 \mathrm{~kb}$ of genomic DNA, encodes a copper-transporting Ptype ATPase [5]. Over 380 mutations in the WD gene have now been identified from a multitude of populations [6] (http://www.medicalgenetics.med.ualberta.ca/wilson/index.p hp). Screening for mutations can thus be laborious, time consuming, and expensive. Since selected mutations appear to be restricted to specific populations [7], establishing the most common mutations in a given population may simplify initial efforts to identify WD gene mutations in specific families.

To date, two large studies have analyzed WD patients from North America. Tanzi et al. [8] studied 50 unrelated families and identified four mutations, though they only screened part of the WD gene. Shah et al. [9] studied 128 North American patients and found that the H1069Q was the most common mutations in the WD gene. In this study, we analyzed 108 unrelated American patients; we find that most

*Address correspondence to this author at the Departments of Molecular and Integrative Physiology, 7629 Medical Sciences II Box 5622, 1301 East Catherine Street, Ann Arbor, MI 48109-5622, USA; E-mail: borjigin@umich.edu mutations are located within exons 14 and 18 and describe five new mutations in the WD gene.

\section{SUBJECTS AND METHODS}

This study included 108 unrelated subjects from the United States with confirmed WD. The diagnosis of Wilson disease was confirmed by a combination of a variety of clinical symptoms, biochemical tests, and detection of gene mutations, which are examined by a group of doctors headed by Dr. Brewer at University of Michigan. Mutation analysis was performed on DNA samples isolated from patient blood samples by PCR amplification, purification, and sequencing of all 21 exons from each of the patients. The primer design and use were based on previously published data [10]. When two different mutations were found in the same exon from the same patient, the PCR products were subcloned and individual clones were sequenced to confirm that they occurred on different chromosomes (demonstrating that the patients were compound heterozygotes). In addition, 98 normal genomic DNA samples from White Americans were analyzed as controls to confirm the absence of the novel ATP7B mutations we found in this study.

\section{RESULTS AND DISCUSSION}

We sequenced ATP7B gene on 216 chromosomes from 108 American WD patients and found mutations in 128 of these. There was a remarkable clustering of mutations in exons 14 and 18 (93 and 14 of 128 mutations, respectively); these two exons account for $84 \%$ of the total number of hits in the ATP7B gene.

We were able to identify both ATP7B mutations in 47 (44\%) of the American patients (Table 1). H1069Q repre- 
Table 1. Mutations Identified in American WD Patients

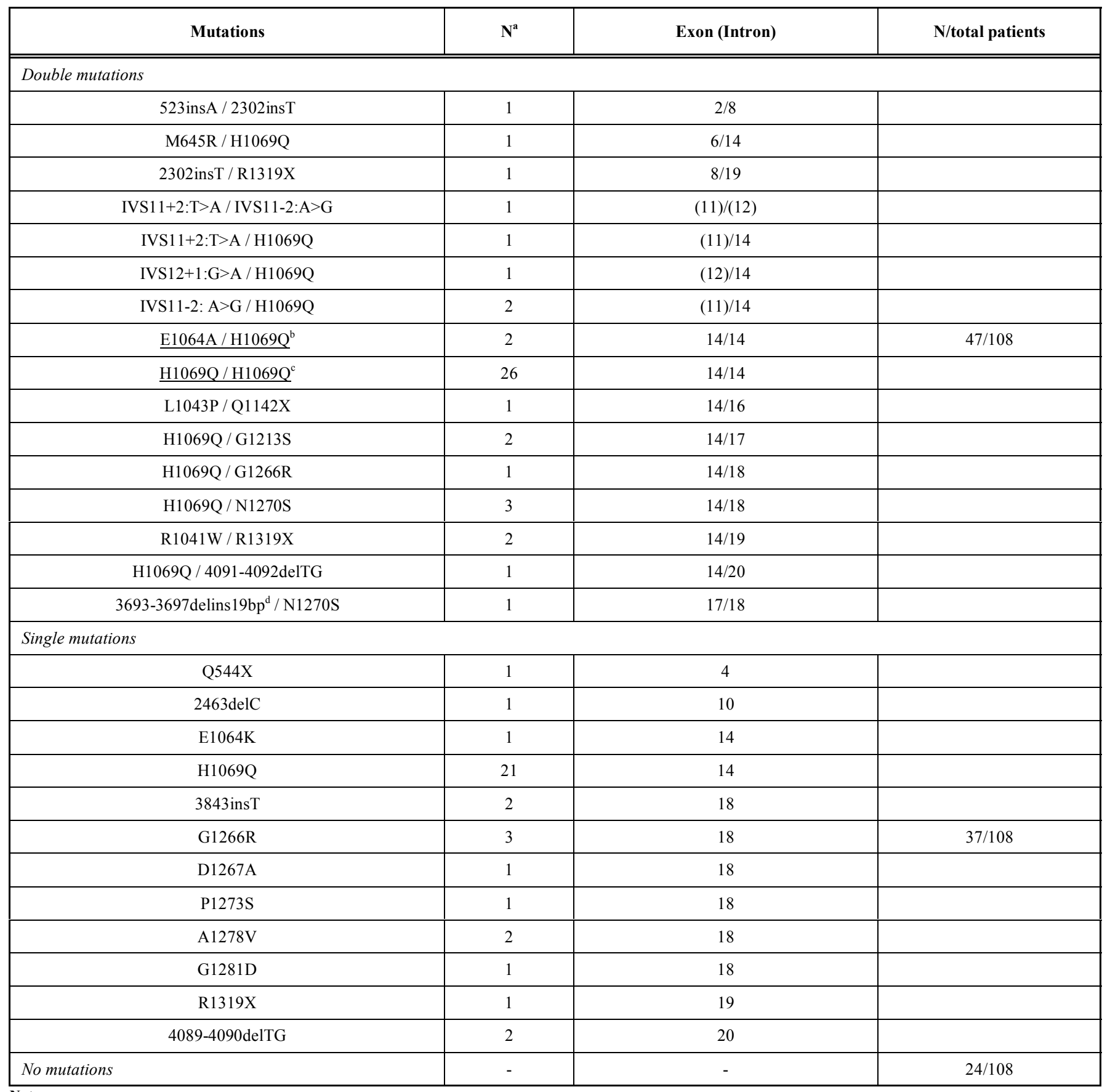

Notes:

a. Number of patients.

b. Confirmed compound heterozygous.

c. Homozygous.

d. TCTGGTACATTAACATTAA

sented the most common mutation found in this cohort. Twenty-six (24\%) patients were homozygous for H1069Q, and two patients were confirmed compound heterozygous for E1064A/H1069Q (by subcloning and sequencing exon 14). The frequent occurrence of $\mathrm{H} 1069 \mathrm{Q}$ in Caucasian patients with WD, including those in the United States [9], is well documented [7].

N1270S was the second most commonly identified mutation in our study, occurring in four of 108 patients
(Tables 1 and 2). Most populations have distinct mutations that are relatively specific for the group $[7,11]$. H1069Q, for instance, is found exclusively in patients of European origin [7]. Conversely, R778G mutation is extremely common for Asians [12]. In addition to these two common mutations, many other rare mutations tend to have regional clustering [7]. N1270S, however, is distinguished by its common occurrence in a large number of groups, including our American cohort, Chinese [12, 13], Korean [14, 15], Japanese [16], Indian [17], Sicilian [8], Bulgarian [18], 
Table 2. Characteristics of the ATP7B Variants Identified

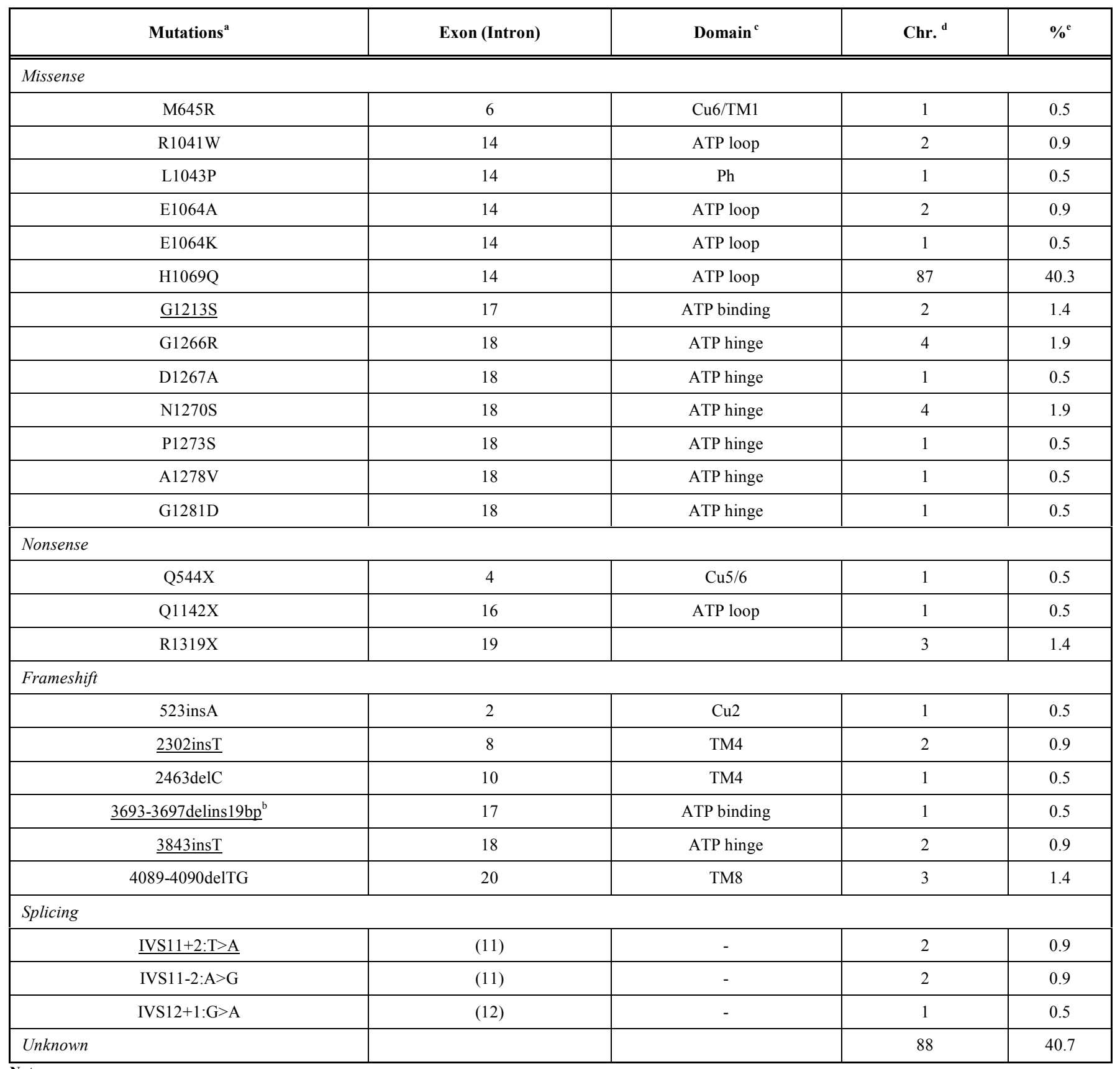

a. Novel mutations found in this study are underlined.

b. TCTGGTACATTAACATTAA.

c. $\mathrm{Cu}=$ Copper binding, $\mathrm{TM}=$ Transmembrane, $\mathrm{Ph}=$ phosphorylation domains.

d. Number of chromosomes affected.

e. Frequency of affected WD chromosomes

Egyptian [19], Brazilian [20], Italian and Turkish [21], and American [9] groups. The frequency of this mutation among diverse ethnic groups suggests that this residue may represent a hot spot for mutation.

In 37 of 108 patients (34\%), we could identify only one mutation in coding regions of ATP7B, while in 24 patients $(22 \%)$, we did not detect any mutations in ATP7B (Table 1). Several groups have reported WD patients without coding mutations in ATP7B [14, 22, 23]. The high frequency of normal ATP7B coding alleles in this large WD cohort and other studies suggests that other mechanisms besides coding mutations in ATP7B could account for a significant number of WD cases. Several possibilities could account for this: 1) presence of a different gene for Wilson disease, 2) mutations in the introns distal from the splice site resulting in altered splicing, 3) mutations in gene regulatory regions resulting in down-regulation of ATP7B gene expression.

Upon comparison of our results with the WD database (http:/www.wilsondisease.med.ualberta.ca/database.aspdatab ase), several novel mutations were identified: two (2302insT 
and 3843insT) were single nucleotide insertions which produce frame shifts in exons 8 and 18; one was a mutation in the conserved intron 11 splice site (IVS11+2:T>A); one was a complex change involving a two nucleotide deletion and insertion of 19 bases (TCTGGTACATTAACATTAA) within exon 17 (3693-3697delins19bp), which causes a frame shift. These four novel mutations lead to gross alterations of affected ATP7B structure and are almost certainly disease-causing mutations.

Finally, we identified G1213S in exon 17. G1213 is located in the conserved ATP7B ATP binding pocket. G1213S results in the insertion of a larger, more hydrophilic residue in the pocket that could alter ATP binding and decrease catalytic function. A similar mutation G1213V has been identified in the Kurdish population and was postulated to be a disease-causing mutation [24]. In addition, an analysis of 98 normal genomic DNA samples, generously provided by Dr. Jeremy Nathans, failed to identify the G1213S variant. Combined, these results suggest that the G1213S mutation may be a disease-causing mutation of ATP7B.

In summary, we present one of the largest North American studies of ATP7B in Wilson disease to date. This study confirms the wide range of mutations that lead to this disorders, but also demonstrates that majority of the confirmed mutations in White Americans can be identified through analysis of exons 14 and 18 in American WD suspects. It is hoped that knowledge of these mutations in ATP7B will lead to more accurate information about prognosis and improved genetic testing and counseling. Further functional studies of the mechanisms of these mutations are warranted.

\section{ACKNOWLEDGEMENTS}

This work was supported by the Department of Embryology at the Carnegie Institution of Washington. We thank Dr. Jeremy Nathans for providing normal genomic DNA samples.

\section{DISCLOSURE STATEMENT}

No competing financial interests exist.

\section{REFERENCES}

[1] Brewer GJ. Wilson's Disease: A Clinician's Guide to Recognition, Diagnosis, and Management. Boston, MA: Kluwer Academis Publishers 2001

[2] Das SK, Ray K. Wilson's disease: an update. Nat Clin Pract Neurol 2006; 2: 482-93.

[3] Pfeiffer RF. Wilson's disease. Semin Neurol 2007; 27: 123-32.

[4] Brewer GJ. Recognition, diagnosis, and management of Wilson's disease. Proc Soc Exp Biol Med 2000; 223: 39-46.
[5] Bull PC, Thomas GR, Rommens JM, Forbes JR, Cox DW. The Wilson disease gene is a putative copper transporting P-type ATPase similar to the Menkes gene. Nat Genet 1993; 5: 344-50

[6] Davies LP, Macintyre G, Cox DW. New mutations in the Wilson disease gene, ATP7B: implications for molecular testing. Genet Test 2008; 12: 139-45.

[7] Ferenci P. Regional distribution of mutations of the ATP7B gene in patients with Wilson disease: impact on genetic testing. Hum Genet 2006; 120: 151-9.

[8] Tanzi RE, Petrukhin K, Chernov I, et al. The Wilson disease gene is a copper transporting ATPase with homology to the Menkes disease gene. Nat Genet 1993; 5: 344-50.

[9] Shah AB, Chernov I, Zhang HT, et al. Identification and analysis of mutations in the Wilson disease gene (ATP7B): population frequencies, genotype-phenotype correlation, and functional analyses. Am J Hum Genet 1997; 61: 317-28.

[10] Petrukhin K, Lutsenko S, Chernov I, et al. Characterization of the Wilson disease gene encoding a P-type copper transporting ATPase: genomic organization, alternative splicing, and structure/function predictions. Hum Mol Genet 1994; 3: 1647-56.

[11] Chappuis P, Callebert J, Quignon V, et al. Late neurological presentations of Wilson disease patients in French population and identification of 8 novel mutations in the ATP7B gene. J Trace Elem Med Biol 2007; 21: 37-42.

[12] Mak CM, Lam CW, Tam S, et al. Mutational analysis of 65 Wilson disease patients in Hong Kong Chinese: identification of 17 novel mutations and its genetic heterogeneity. J Hum Genet 2008; 53: 5563.

[13] Wan L, Tsai CH, Tsai Y, et al. Mutation analysis of Taiwanese Wilson disease patients. Biochem Biophys Res Commun 2006; 345: 734-8.

[14] Yoo HW. Identification of novel mutations and the three most common mutations in the human ATP7B gene of Korean patients with Wilson disease. Genet Med 2002; 4: 43S-8S.

[15] Park S, Park JY, Kim GH, et al. Identification of novel ATP7B gene mutations and their functional roles in Korean patients with Wilson disease. Hum Mutat 2007; 28: 1108-13.

[16] Yamaguchi A, Matsuura A, Arashima S, et al. Mutations of ATP7B gene in Wilson disease in Japan: identification of nine mutations and lack of clear founder effect in a Japanese population. Hum Mutat 1998; (Suppl 1): S320-2.

[17] Santhosh S, Shaji RV, Eapen CE, et al. ATP7B mutations in families in a predominantly Southern Indian cohort of Wilson's disease patients. Indian J Gastroenterol 2006; 25: 277-82.

[18] Todorov T, Savov A, Jelev H, et al. Spectrum of mutations in the Wilson diease gene (ATP7B) in the Bulgarian population. Clin Genet 2005; 68: 474-76.

[19] Abdelghaffar TY, Elsayed SM, Elsobky E, et al. Mutational analysis of ATP7B gene in Egyptian children with Wilson disease: 12 novel mutations. J Hum Genet 2008; 53: 681-7.

[20] Deguti MM, Genschel J, Cancado EL, et al. Wilson disease: novel mutations in the ATP7B gene and clinical correlation in Brazilian patients. Hum Mutat 2004; 23: 398 .

[21] Figus A, Angius A, Loudianos G, et al. Molecular pathology and haplotype analysis of Wilson disease in Mediterranean populations. Am J Hum Genet 1995; 57: 1318-24.

[22] Vrabelova S, Letocha O, Borsky M, et al. Mutation analysis of the ATP7B gene and genotype/phenotype correlation in 227 patients with Wilson disease. Mol Genet Metab 2005; 86: 277-85.

[23] Loudianos G, Dessi V, Angius A, et al. Wilson disease mutations associated with uncommon haplotypes in Mediterranean patients. Hum Genet 1996; 98: 640-2.

[24] Kalinsky H, Funes A, Zeldin A, et al. Novel ATP7B mutations causing Wilson disease in several Israeli ethnic groups. Hum Mutat 1998; 11: 145-51. 